\title{
Ion-implantation induced anomalous surface amorphization in silicon
}

\author{
T. Lohner ${ }^{\text {a,* }}$, E. Kótai ${ }^{\text {a }}$, N.Q. Khánh ${ }^{\text {a }}$, Z. Tóth ${ }^{b}$, M. Fried ${ }^{\text {a }}$, K. Vedam ${ }^{\text {c }}$, N.V. Nguyen ${ }^{\text {c }}$, \\ L.J. Hanekamp ${ }^{\text {d }}$ A. van Silfhout ${ }^{d}$ \\ a Joint Chair for Fxperimental Physics of Technical University Budapest and KFKI Research Institute for Materials Science, P.O.B. 49, \\ H-1525 Budapest, Hungary \\ ${ }^{b}$ TUNGSRAM Co. Ltd., Bródy I. Research Center, Váci út 77., H-1340 Budapest, IV., Hungary \\ c Materials Research Laboratory, Pennsylvania State University, University Park, PA 16802, USA \\ ${ }^{d}$ Faculty of Applied Physics, University of Twente, P.O.B. 217, 7500 AE Enschede, The Netherlands
}

Spectroscopic ellipsometry (SE), high-depth-resolution Rutherford backscattering (RBS) and channeling have been used to examine the surface damage formed by room temperature $\mathrm{N}$ and $\mathrm{B}$ implantation into silicon. For the analysis of the $\mathrm{SE}$ data we used the conventional method of assuming appropriate optical models and fitting the model parameters (layer thicknesses and volume fraction of the amorphous silicon component in the layers) by linear regression. The dependence of the thickness of the surface-damaged silicon layer (beneath the native oxide layer) on the implantation parameters was determined: the higher the dose, the thicker the disordered layer at the surface. The mechanism of the surface amorphization process is explained in relation to the ion beam induced layer-by-layer amorphization. The results demonstrate the applicability of spectroscopic ellipsometry with a proper optical model. RBS, as an independent cross-checking method supported the constructed optical model.

\section{Introduction}

The motion of crystalline-amorphous (c-a) silicon interfaces under ion beam irradation has been a subject of considerable interest in the last years [1-5]. A pre-existing planar c-a interface submitted to ion irradiation can move either towards the amorphous side (crystallization regime) or towards the crystalline side (amorphization regime) depending on-the substrate temperature, the irradiating beam, the dose rate, the substrate orientation and the impurity content in the layer. Most of the work was performed with heavy ion implantation [1-5], where this effect was more pronounced than in the case of light ions.

A similar effect of anomalous surface disordering was observed in some other cases [6-8]. Here anomalous means that this disorder is far from that in the depth of the projected range. Moreover, there is no pre-existing c-a interface before ion irradiation. Earlier we have shown this phenomenon in the case of boron implanted silicon by the combined application of single-wave length ellipsometry and high-depth-resolution Rutherford backscattering spectrometry $[9,10]$. However, in the case of low energy implantation the dam-

\footnotetext{
* Corresponding author, phone +36 11699499 , fax +36 11550694.
}

age region is very close to the surface, thus single-wave length ellipsometry is not able to distinguish the surface and the mean projected range defects. In this case spectroscopic ellipsometry is a very helpful tool to investigate such a phenomenon, because it is very sensitive to the surface disorder.

The applicability of SE for the nondestructive determination of ion-implantation induced damage profiles was demonstrated in detail by several research groups [6,7,11-13]. In the present paper we report on an RBS and SE study of light ion $\left(\mathrm{N}^{+}\right.$and $\left.\mathrm{B}^{+}\right)$ implantation induced anomalous surface amorphization in silicon.

\section{Experimental}

To investigate the surface amorphization process $200 \mathrm{keV} \mathrm{N}^{+}$or $10 \mathrm{keV} \mathrm{B}^{+}$ions were implanted at room temperature in $\langle 100\rangle$ silicon. The implantation was performed into Wacker single-crystal silicon at the Central Research Institute for Physics, Budapest. The implantation was done at room temperature with a current density of $190 \mathrm{nA} / \mathrm{cm}^{2}$ for nitrogen and 0.5 $\mathrm{mA} / \mathrm{cm}^{2}$ for boron. The SE spectra were obtained partly on a SOPRA rotating polarizer ellipsometer in the $1.5-4.5 \mathrm{eV}$ photon energy region at the Pennsylvania State University ( $\mathrm{N}$ implantation), and partly at 

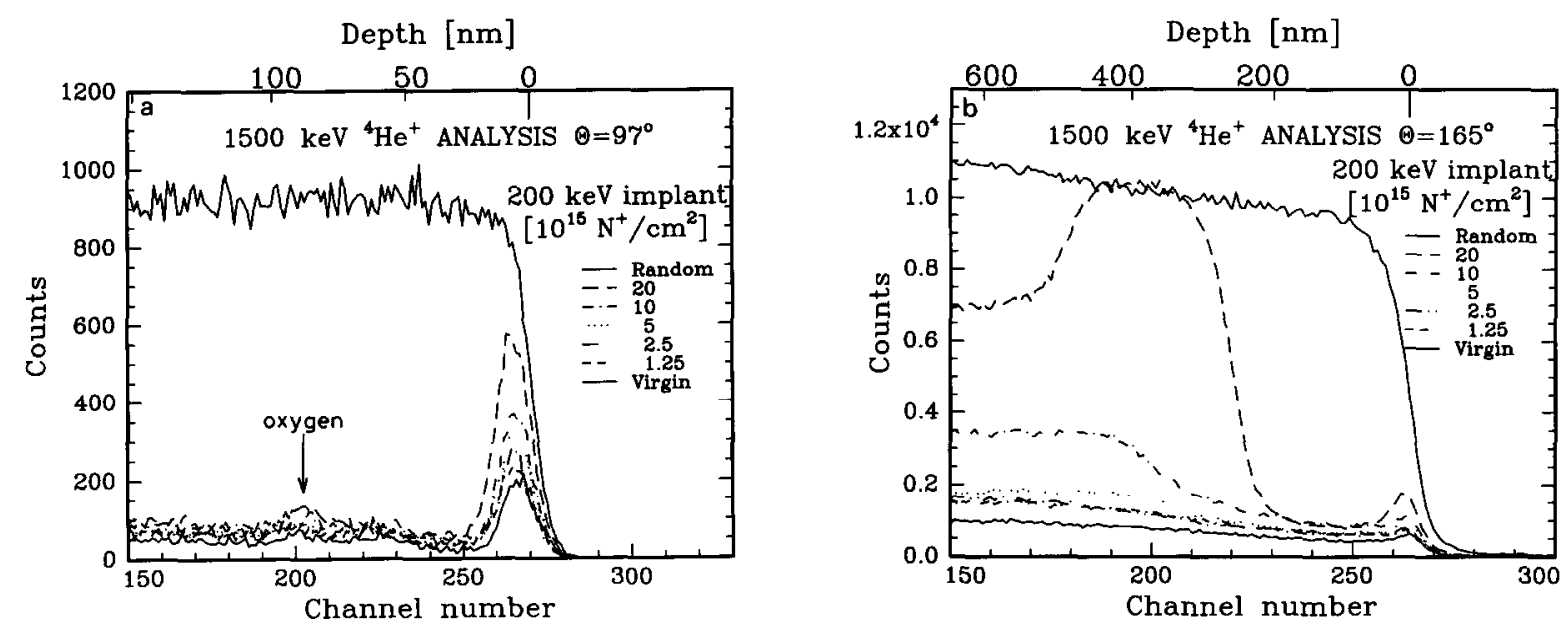

Fig. 1(a). High-depth resolution RBS spectra for the series of nitrogen-implanted samples recorded with the detector placed at $97^{\circ}$ scattering angle. (b) Normal resolution spectra recorded with the detector placed at $165^{\circ}$ scattering angle.

Twente University in the range of $300-650 \mathrm{~nm}$ with a rotating analyzer ellipsometer.

Rutherford backscattering and channeling techniques with $1.5 \mathrm{MeV} \mathrm{He}{ }^{+}$ions were used to determine the radiation damage. The detector was placed to detect ions scattered through $97^{\circ}$ (i.e. with a glancing exit angle of $7^{\circ}$ to the surface). In this geometry, the depth resolution was better than $5 \mathrm{~nm}$ [14]. To evaluate the spectra we used the RBX program written by Kótai [15], which can also handle channeled spectra. The thicknesses of the surface oxide and disordered layers were deduced from the oxygen pcak (ch. No. 201) and the damage peak of the spectra in Fig. 1a, respectively. The underlying damage level (i.e. the defect volume fraction) was deduced from spectra in Fig. $1 b$.

\section{Results and discussion}

\section{1. $200 \mathrm{keV}$ nitrogen}

In this case the optical model consists of a native oxide layer, a thin amorphous silicon layer and a slightly damaged semiinfinite layer. For the evaluation the 400 $\mathrm{nm}$ wavelength was chosen as an upper limit because in this case the optical penetration depth (even in crystalline $\mathrm{Si}$ ) is not larger than $200 \mathrm{~nm}$.

For the analysis of the spectroscopic ellipsometry data we used the conventional method of assuming appropriate optical models and filting the model parameters (layer thicknesses and volume fractions of the amorphous silicon component in the layers) by linear regression. The data of the nitrogen-implanted samples were analyzed using a FORTRAN program developed at the Pennsylvania State University. It is essential to choose a proper starting value for model parameters, otherwise the program may find a false minimum. We used grid search before fitting. It is important that we used the complex dielectric function of implanted amorphous silicon [16]. The goodness of the fit is estimated by the unbiased estimator:

$$
\begin{aligned}
\sigma= & \frac{1}{2 N-p-1}\left\{\sum _ { j = 1 } ^ { N } \left[\left(\cos \Delta_{j}^{\text {exp }}-\cos \Delta_{j}^{\text {calc }}\right)^{2}\right.\right. \\
& \left.\left.+\left(\tan \Psi_{j}^{\text {exp }}-\tan \Psi_{j}^{\text {calc }}\right)^{2}\right]\right\}^{1 / 2},
\end{aligned}
$$

where $N$ is the number of wavelengths and $p$ is the number of fitted parameters.

Figs. $1 \mathrm{a}$ and $1 \mathrm{~b}$ present the high-depth resolution RBS spectra recorded with a detector placed at scattering angle of $97^{\circ}$ and spectra recorded with a detector placed at scattering angle of $165^{\circ}$, respectively, for the series of nitrogen-implanted samples. In accordance with projected range calculations, significant buried disorder is observable around $400 \mathrm{~nm}$ depth (Fig. 1b). Surprisingly, the energetic nitrogen ions are shown to have created a well defined disorder at the surface of silicon. The higher the dose, the larger the disorder peak at the surface and the higher the damage level in the first $200 \mathrm{~nm}$. To study this anomalous surface disorder (assumed thin amorphous layer) SE is extremely useful because of its sensitivity.

Measured ellipsometric spectra of silicon samples implanted with different doses of nitrogen ions together with the results of multiparameter fittings are 


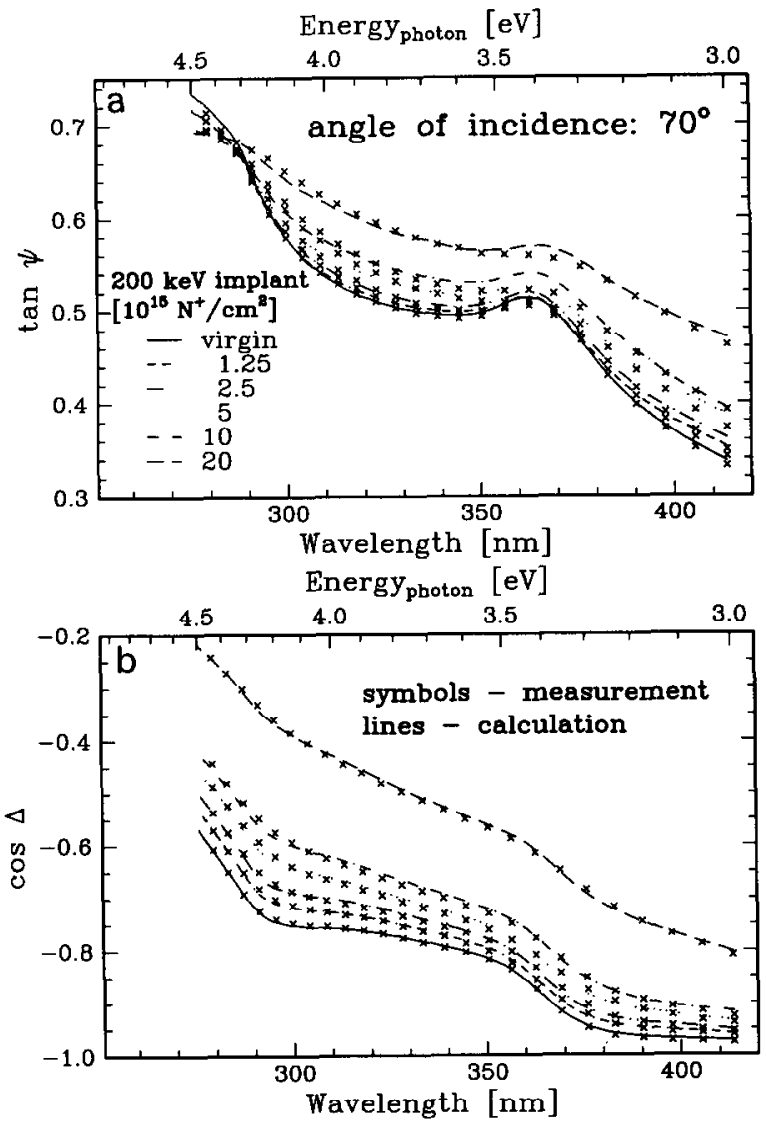

Fig. 2. Results of SE fitting for the nitrogen-implanted silicon samples. The angle of incidence was $70^{\circ}$.

shown in Fig. 2. For comparison, a reference spectrum of virgin (unimplanted) silicon is also presented.

Table I summarizes the layer thickness values and damage levels resulting from the evaluation of SE and RBS measurements. For SE evaluation the complex dielectric function of ion-implantation induced amorphous silicon [16] was used. For RBS analysis we used the density of silicon $\left(5 \mathrm{e} 22\right.$ atoms $\left./ \mathrm{cm}^{3}\right)$ to calculate the thickness of the surface disorder layer. Comparing the thickness values of the surface amorphous layer obtained by different methods one can establish that they are in very good agreement. Moreover, SE evaluation using the volume fraction of the amorphous silicon component in the damaged surface layer as a free parameter yielded $115 \% \pm 8 \%$ for the case of highest dose implanted sample. This fact proves that this thin layer is amorphous indeed.

The other parameters (native oxide thickness and background damage level) show systematic differences between the two methods. This reveals that SE is sensitive to these effects, but for precise evaluation an adequate optical model is needed. The systematic dif- ference betwecn the background damage levels can be explained by the nature of this damage. In the optical model we use the complex dielectric function of amorphous silicon as reference. For light ions the normal damage consists mainly of point defects which seem to change the dielectric function in a larger volume than their atomic volume. (Point defects cause strain in the lattice displacing the neighbouring atoms.) RBS determines the amount of the defects (displaced atoms $/ \mathrm{cm}^{3}$ ) but SE senses the volume of the optically changed (stressed) lattice.

The difference in the thickness of the surface (native) oxide can be explained in a like manner. We use the refractive index of $\mathrm{SiO}_{2}$ but this surface is possibly roughened and unstochiometric. SE determines an effective thickness but RBS gives atoms $/ \mathrm{cm}^{2}$.

\section{2. $10 \mathrm{keV}$ boron}

In this case the buried disorder peak is situated in the vicinity of the surface disorder because of the shorter projected range, thus complicating the analysis. To take into account this fact one should apply the optical model developed earlier for damage depth pro-

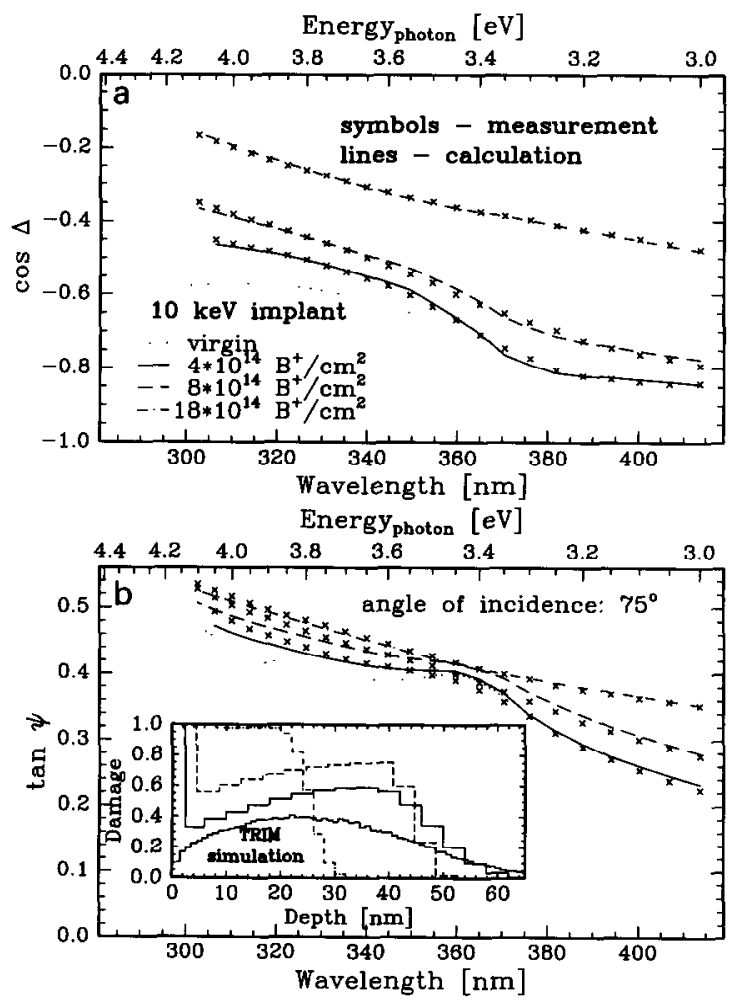

Fig. 3. Results for SE fitting for the $10 \mathrm{keV}$ boron implanted samples. The insert shows the damage profile deduced from the SE fitting together with the result of a TRIM simulation. 
Table 1

Implantation conditions together with the results of SE fitting procedure and RBS/channeling analysis. Nitrogen was implanted with $200 \mathrm{keV}$, boron with $10 \mathrm{keV}$. $D_{\mathrm{a}-\mathrm{Si}}$ is the thickness of the surface amorphous silicon, $D_{\mathrm{SiO}_{2}}$ is the thickness of native oxide, $\sigma$ is the unbiased estimator for SE. Damage level means the volume fraction of defect

\begin{tabular}{|c|c|c|c|c|c|c|c|c|}
\hline \multirow[t]{2}{*}{ Ion } & \multirow{2}{*}{$\begin{array}{l}\text { Dose } \\
{\left[10^{14} / \mathrm{cm}^{2}\right]}\end{array}$} & \multicolumn{2}{|l|}{$D_{\mathrm{a}-\mathrm{Si}}[\mathrm{nm}]$} & \multicolumn{2}{|c|}{ Damage level [\%] } & \multicolumn{2}{|c|}{$D_{\mathrm{SiO}_{2}}[\mathrm{~nm}]$} & \multirow[t]{2}{*}{$\sigma\left[10^{-2}\right]$} \\
\hline & & $\mathrm{SE}$ & RBS & $\overline{S E}$ & RBS & $\overline{\mathrm{SE}}$ & RBS & \\
\hline $\mathrm{N}^{+}$ & 12 & $0.7 \pm 0.1$ & $0.4 \pm 0.1$ & $8 \pm 2$ & $2.5 \pm 0.2$ & $2.0 \pm 0.1$ & $1.0 \pm 0.3$ & 0.62 \\
\hline $\mathbf{N}^{+}$ & 25 & $1.0 \pm 0.2$ & $1.4 \pm 0.2$ & $9 \pm 2$ & $2.8 \pm 0.2$ & $2.2 \pm 0.2$ & $1.1 \pm 0.3$ & 0.91 \\
\hline $\mathbf{N}^{+}$ & 50 & $2.3 \pm 0.2$ & $1.9 \pm 0.2$ & $13 \pm 3$ & $3.0 \pm 0.3$ & $2.0 \pm 0.2$ & $1.1 \pm 0.3$ & 1.25 \\
\hline $\mathrm{N}^{+}$ & 100 & $3.1 \pm 0.2$ & $2.4 \pm 0.2$ & $11 \pm 3$ & $5.2 \pm 0.4$ & $2.3 \pm 0.2$ & $1.5 \pm 0.3$ & 1.04 \\
\hline $\mathrm{N}^{+}$ & 200 & $7.0 \pm 0.3$ & $7.4 \pm 0.3$ & $14 \pm 3$ & $5.7 \pm 0.4$ & $4.8 \pm 0.2$ & $2.3 \pm 0.4$ & 0.79 \\
\hline $\mathbf{B}^{+}$ & 4 & $1.9 \pm 0.4$ & - & Fig. 3 & - & $1.5 \pm 0.2$ & & 0.43 \\
\hline $\mathrm{B}^{+}$ & 8 & $4.5 \pm 0.8$ & - & Fig. 3 & - & $2.0 \pm 0.2$ & & 0.52 \\
\hline $\mathrm{B}^{+}$ & 18 & $26 \pm 2$ & - & Fig. 3 & - & $2.2 \pm 0.2$ & & 0.39 \\
\hline
\end{tabular}

filing [13]. This optical model consists of a stack of films with fixed and equal thicknesses and damage levels determined by a depth profile function of coupled half-Gaussians. The model uses five parameters: the center, the height, and two standard deviations of the profile, as well as the thickness of the native oxidc.

For the present situation this model was enhanced with an added surface damaged layer (beneath the native oxide) which is treated independently from the buried damage profile. The measured ellipsometric parameters in the UV region (above $3.5 \mathrm{eV}$ where the optical absorption is high) contain information dominantly on the surface layer. At longer wavelengths the optical penetration depth is larger and consequently the ellipsometric parameters in this range provide information mainly from the deeper region.

The results of the "best fit" together with the experimental spectra are shown in Fig. 3. The insert illustrates the damage distribution extracted from the fitting procedure together with a Monte Carlo simulation (TRIM code [17]). The nominal values of the layer thicknesses are presented in Table 1.

The mechanism of the surface disordering process will be discussed in relation to ion beam induced layer-by-layer disordering at the silicon-native oxide interface elsewhere [18].

\section{Conclusion}

Spectroscopic ellipsometry, high-depth-resolution Rutherford backscattering and channeling have been used to examine the surface damage formed by room temperature $\mathrm{B}$ and $\mathrm{N}$ implantation into silicon. $\mathrm{A}$ multiparameter fitting procedure of ellipsometric data was applied to evaluate the damage depth profile.

It was found that the thickness of the surfacedamaged silicon layer (beneath the native oxide layer) increased monotonously with increasing implantation dose.

The results demonstrate the applicability of spectroscopic ellipsometry with a proper optical model. RBS, as an independent cross-checking method, basically supportcd the constructed optical model.

\section{Acknowledgements}

One of the authors (T.L.) is grateful to IREX for a scholarship and wishes to thank colleagues at the Pennsylvania State University for collaboration during his stay there. One of the authors (M.F.) is grateful to Twente University for a scholarship during his research work. Partial support from OTKA grants (No. 3265, No. 1829 and No. F4378) is greatly appreciated.

\section{References}

[1] F. Priolo and E. Rimini, Mater. Sci. Rep. 5 (1990) 319.

[2] J.S. Williams, R.G. Elliman, W.L. Brown and T.E. Seidel, Phys. Rev. Lett. 55 (1985) 1482.

[3] J. Linnros, G. Holmen and B. Svensson, Phys. Rev. B 32 (1985) 2770.

[4] A. Battaglia, F. Priolo and E. Rimini, Appl. Surf. Sci. 56-58 (1992) 577.

[5] J. Nakata, Phys. Rev. B 43 (1991) 14643.

[6] M. Erman and J.B. Theeten, Surf. Interface Anal. 4 (1982) 98.

[7] M. Erman and J.B. Theeten, Surf. Sci. 135 (1983) 353.

[8] G.Q. Yang, N.Q. Khanh, M. Fried, E. Kótai, V. Schiller, L.C. Lu, J. Gyulai and S.C. Zou, Radiat. Eff. Def. Sol. 115 (1990) 183.

[9] T. Lohner, G. Mezey, E. Kótai, F. Pászti, A. Manuaba and J. Gyulai, Nucl. Instr. and Meth. 209/210 (1983) 615.

[10] T. Lohner, E. Kótai, F. Pászti, A. Manuaba, M. Fried and J. Gyulai, J. Radioanal. Nucl. Chem. 83(1) (1984) 75. 
[11] P.J. McMarr, K. Vedam and J. Narayan, J. Appl. Phys. 59 (1986) 694.

[12] J. Vanhellemont, P. Roussel and H.E. Maes, Nucl. Instr. and Meth. B 55(1991) 183.

[13] M. Fried, T. Lohner, W.A.M. Aarnink, LJ. Hanekamp and A. van Silfhout, J. Appl. Phys. 71 (1992) 2835.

[14] G. Mezey, E. Kótai, T. Lohner, T. Nagy, J. Gyulai and A. Manuaba, Nucl. Instr. and Meth. 149 (1978) 235.
[15] E. Kótai, these Proceedings (IBA-11, Balatonfüred, Hungary, 1993), Nucl. Instr. and Meth. B 85 (1994) 588.

[16] M. Fried, T. Lohner, W.A. M Aarnink, L.J. Hanekamp and A. van Silfhout, J. Appl. Phys. 71 (1992) 5260.

[17] J.F, Ziegler, J.P. Biersack, U. Littmark, The Stopping and Range of lons in Solids (Pergamon, New York, 1985).

[18] T. Lohner et al., to be published. 